\title{
Connecting the potential theory to the quantum field theory*
}

\author{
H.Sazdjian \\ Groupe de Physique Théorique, Institut de Physique Nucléaire, \\ Université Paris XI, F-91406 Orsay Cedex, France
}

Received March 12, 1998

\begin{abstract}
A constraint theory is applied to two-particle systems to construct Poincaré invariant wave equations with interaction potentials. The latter can be determined in the quantum field theory from Feynman diagrams. Their properties are analyzed in the case of QED. The constraint theory provides a consistent means for solving the Bethe-Salpeter equation with covariant propagators.
\end{abstract}

Key words: relativistic wave equations, Bethe-Salpeter equation, bound states, constraint theory

PACS: 03.65.Pm, 11.10.St, 11.80.Fv

The idea of describing relativistic systems of a finite number of interacting particles with a finite number of degrees of freedom, as long as radiation and inelasticity effects are ignored, is a natural generalization of the approaches used in nonrelativistic mechanics [1]. It is understood that the Galilei invariance of the nonrelativistic theory should now be replaced by the Poincaré invariance. Pioneering works in this domain were realized by Wheeler and Feynman [2], using a Fokker type action, and by Dirac [3], using a Hamiltonian formalism.

Fokker type actions, while ensuring by construction a manifest covariance, had the main defect of leading to integro-differential equations in individual particle proper times that were very difficult to reconcile with initial value problems. Furthermore, relative times, like the space-like coordinates, play there a dynamical role. This approach was later amended by Bel and replaced by a manifestly covariant Newtonian formalism in which particle position four-vectors satisfy secondorder differential equations with respect to proper times. The reparametrization invariance of world lines was guaranteed by the appropriate conditions, called predictivity conditions, which are imposed on the relativistic forces for the nonlinear equations to be satisfied [4].

The requirement that relative times should play only the role of evolution

${ }^{*}$ Dedicated to the 70th anniversary of Professor Roman Gaida. 
parameters also makes possible the description of the system with a single time parameter, common to all particles, and chosen on any space-like surface. In this case the starting point can be represented by a Lagrangian with three-dimensional variables. Here, however, it is the implementation of the Poincaré invariance that becomes a difficult task. The corresponding Lagrangian actually becomes, in the interacting case, dependent on derivatives of an infinite order. This line of approach was developed by Gaida and his collaborators [5].

The Lagrangian approach can also be developed in a manifestly covariant formalism. As in the one-particle case, the Lagrangian belongs to the category of singular Lagrangians as a consequence of the reparametrization invariance of the theory with respect to definitions of individual times $[6,7]$. A connection can also be established between these two Lagrangian approaches [8].

In the present article I shall focus on some developments of the line of approach based on the Hamiltonian formalism. Using a single time formalism, Bakamjian and Thomas [9] and Foldy [10] developed perturbative realizations of the Poincaré algebra which manifests itself through nonlinear equations. However, it was soon realized at the classical level that it was impossible to identify canonical coordinates with position variables $[11,12]$. The latter had to be constructed as (complicated) dynamical variables by means of their covariance properties [13]. This means that the Hamiltonian approach is not well suited for the construction of world lines. However, it becomes a powerful tool at the quantum level, where world lines do not play a fundamental role. Here, the priority goes towards the realization of the Poincaré invariance and the construction of the Hilbert space of states.

The major progress in this approach came from the constraint theory developed earlier in its general lines by Dirac $[14,15]$. The constraint theory allows the elimination of the redundant variables without necessarily breaking the manifest symmetries of the initial theory. Thus, it was possible through appropriate constraints to eliminate, for interacting particle systems, the relative energies, or the relative times, without destroying the manifest covariance. The Poincaré invariance of the theory is realized in the presence of the constraints by means of the Dirac brackets. The constraint theory approach was first applied to interacting particle systems by Droz-Vincent [16], then by Todorov [17] and Komar [18]. The separability (cluster decomposition) condition was also shown to have formal or perturbative realizations [19-23]. The problem of constructing the Dirac observables in the presence of classical fields (Abelian or non-Abelian) was later investigated by Lusanna and collaborators [24]

The classical theory is quantized rather easily in the two-particle case [25-29]. For definiteness, a two-fermion system can be described by the following two wave equations which are generalizations of the Dirac equation [28]:

$$
\begin{aligned}
& \left(\gamma_{1} \cdot p_{1}-m_{1}\right) \widetilde{\Psi}=\left(\gamma_{2} \cdot p_{2}+m_{2}\right) \widetilde{V} \widetilde{\Psi}, \\
& \left(\gamma_{2} \cdot p_{2}-m_{2}\right) \widetilde{\Psi}=\left(\gamma_{1} \cdot p_{1}+m_{1}\right) \widetilde{V} \widetilde{\Psi},
\end{aligned}
$$

where $\widetilde{\Psi}$ is a spinor wave function of rank two and $\widetilde{V}$ is a Poincaré invariant inter- 
acting potential which in the general case is represented by an integral operator, but in simple approximations can be represented by a multiplicative function. The compatibility condition of the two equations leads to the following constraints:

$$
\begin{aligned}
{\left[\left(p_{1}^{2}-p_{2}^{2}\right)-\left(m_{1}^{2}-m_{2}^{2}\right)\right] \widetilde{\Psi} } & =0 \\
{\left[p_{1}^{2}-p_{2}^{2}, \widetilde{V}\right] \widetilde{\Psi} } & =0 .
\end{aligned}
$$

The first equation is the constraint that allows the elimination of the (covariant) relative energy of the two particles. Introducing the total and relative variables $P=p_{1}+p_{2}, p=\left(p_{1}-p_{2}\right) / 2, x=x_{1}-x_{2}$ and defining transverse and longitudinal components with respect to the total momentum,

$$
q_{\mu}^{\mathrm{T}}=q_{\mu}-\frac{q \cdot P}{P^{2}} P_{\mu}, \quad q_{\mathrm{L}}=q \cdot P / \sqrt{P^{2}}, \quad P_{\mathrm{L}}=\sqrt{P^{2}}, \quad r=\sqrt{-x^{\mathrm{T} 2}},
$$

it can be rewritten as

$$
C(P, p) \equiv 2 P_{\mathrm{L}} p_{\mathrm{L}}-\left(m_{1}^{2}-m_{2}^{2}\right) \approx 0
$$

It also determines the evolution law of the system in the (covariant) relative time $x_{\mathrm{L}}$.

The second equation means that potential $\tilde{V}$ does not depend on the relative time $x_{\mathrm{L}}$ :

$$
\tilde{V}=\tilde{V}\left(x^{\mathrm{T}}, p^{\mathrm{T}}, P_{\mathrm{L}}, \gamma_{1}, \gamma_{2}\right) .
$$

The elimination of the relative energy and relative time from the wave equations allows one to bring the final eigenvalue equation of the system to a threedimensional Pauli-Schrödinger type equation [30], thus leading to the same quantum numbers as those of nonrelativistic quantum mechanics. This reduction is done without a loss of the Poincaré invariance of the theory.

The structure of potential $\widetilde{V}$ in the wave equations (1) is not fixed by the constraint theory. It can be chosen empirically or phenomenologically according to the nature of the system under study. Wave equations (1), in a different representation, were extensively used by Crater and Van Alstine and collaborators in spectroscopic calculations for quarkonium, positronium and muonium systems [31].

Generalizations of the above approach to the case of systems including more than two particles were also considered [32-34].

Wave equations (1) can thus be used for practical calculations related to twoparticle systems. They define a relativistic framework for the potential theory. However, more can be said about potential $\widetilde{V}$ : it can be calculated in the perturbation theory from Feynman diagrams. The reason for this is related to the fact that the constraint theory can also be applied to equations that result from the quantum field theory. Since one expects from relative times not to play a dynamical role, one can impose constraint (5) on the Bethe-Salpeter equation [35] or on the integral equation of the two-particle Green function of the quantum field theory, in 
QED, for instance. Expanding the above equations around the constraint hypersurface (5) and rearranging terms one ends up for the two-particle system with wave equations that have the same structure as equations (1), with $\widetilde{V}$ determined in terms of the off-mass shell scattering amplitude through the Lippmann-Schwinger type equation [36]:

$$
\widetilde{V}=\widetilde{T}\left(1-\widetilde{g}_{0} \widetilde{T}\right)^{-1}, \quad \widetilde{T}\left(P, p, p^{\prime}\right)=\left.\frac{\mathrm{i}}{2 P_{\mathrm{L}}} T\left(P, p, p^{\prime}\right)\right|_{C(P, p), C\left(P, p^{\prime}\right)},
$$

where $T$ is the off-mass shell scattering amplitude and $\widetilde{T}$ is defined from it by the imposition of constraint $C$ [equation (5)] on its external particle momenta. The operator $\widetilde{g}_{0}$ is related to the free two-particle Green function in the presence of constraint $C$ :

$$
\begin{aligned}
\widetilde{g}_{0} & =\left.\frac{\mathrm{i}}{\left(\gamma_{1} \cdot p_{1}-m_{1}+\mathrm{i} \varepsilon\right)} \frac{\mathrm{i}}{\left(\gamma_{2} \cdot p_{2}-m_{2}+\mathrm{i} \varepsilon\right)}\left(p_{1}^{2}-m_{1}^{2}\right)\right|_{C(P, p)} \\
& =\left.\frac{\mathrm{i}}{\left(\gamma_{1} \cdot p_{1}-m_{1}+\mathrm{i} \varepsilon\right)} \frac{\mathrm{i}}{\left(\gamma_{2} \cdot p_{2}-m_{2}+\mathrm{i} \varepsilon\right)}\left(p_{2}^{2}-m_{2}^{2}\right)\right|_{C(P, p)} .
\end{aligned}
$$

Equation (7) belongs to the general class of relations found in the quasipotential approach $[37,38]$. These differ from each other by the choice of the hypersurface on which the three-dimensional projection operation is done and by the choice of operator $\widetilde{g}_{0}$. The constraint theory can thus be considered as equivalent to the quasipotential approach. By varying the choices of constraint $C$ and operator $\widetilde{g}_{0}$ one can generate all the possible varieties of the quasipotential approach. The choices that were made above seem, however, to possess the optimal properties. We shall now analyze these features.

Equation (7) introduces definite energy and mass dependences in potential $\widetilde{V}$. When one of the particles becomes infinitely massive, equations (1) reduce to the one-particle Dirac equation in the presence of the static potential created by the heavy particle (radiative corrections being neglected). When the two particles become heavy, equations (1) reduce at the leading order to the nonrelativistic Schrödinger equation. The next-to-leading terms yield the $O\left(1 / c^{2}\right)$ corrections. To this order, the resulting Hamiltonian is equivalent to the Breit Hamiltonian [39], which is known to yield the correct $O\left(\alpha^{4}\right)$ corrections in QED spectroscopy, $\alpha$ being the fine structure constant [40].

Equation (7) can also be analyzed in the general case. We observe that the second term in the right-hand side of the first equation yields an iteration series in which the integrations, because of the presence of constraint $C$, are threedimensional. The corresponding terms yield additional diagrams to the ordinary Feynman diagrams; we call them "constraint diagrams". These play a crucial role in building up potential $\tilde{V}$. The constraint diagrams cancel the singularities of the reducible diagrams of the scattering amplitude $\widetilde{T}$, at least in the elastic unitarity region. Potential $\widetilde{V}$ is, therefore, an irreducible kernel, free of singularities in the $s$-channel, leading to correct hermiticity and unitarity properties. A more detailed 
analysis shows that at the second order of the perturbation theory the sum of twophoton exchange diagrams calculated in covariant gauges is free, at the leading order, of spurious infra-red singularities and yields the correct $O\left(\alpha^{4}\right)$ terms of the QED bound state spectroscopy [36]. This is in contrast with the Bethe-Salpeter equation which displays several defects when the perturbation theory with formal powers of the coupling constant is used together with a covariant propagator for the photon. In the nonrelativistic limit, the one-photon exchange diagram yields relativistic corrections of order $1 / c$ instead of $1 / c^{2}$ [41]. In spectroscopic calculations, two-photon exchange diagrams yield spurious infra-red singularities [42]. These effects are cancelled only with the inclusion of higher-order diagrams, a feature that enormously complicates the use of the equation in the perturbation theory. Therefore, the constraint theory can also be viewed as a means of a consistent resolution of the Bethe-Salpeter equation within the framework of the covariant perturbation theory.

The analysis of equation (7) can be continued to higher-order terms. Using a variant of the eikonal approximation adapted to the present problem, the leading term (in the sense of QED infra-red counting rules) of the sum of $n$-photon exchange diagrams can be evaluated and is found to be free of spurious singularities; it yields in three-dimensional $x$-space a local expression proportional to $\left(\alpha /\left(2 P_{\mathrm{L}} r\right)\right)^{n}$. The series of leading terms can be summed and the result is also a local function in $r$, together with a dependence on the c.m. total energy $P_{L}$. In this approximation the expression for the QED potential is, in the Feynman gauge $[36]$ :

$$
\widetilde{V}=\tanh \left(\gamma_{1} \cdot \gamma_{2} V\right), \quad V=\frac{1}{4} \ln \left(1+\frac{2 \alpha}{P_{\mathrm{L}} r}\right)
$$

This potential is equivalent to the one proposed by Todorov in the quasipotential approach on the basis of a minimal substitution rule upon the identification of the motion in the c.m. frame into that of a fictitious particle with reduced mass and energy [38]. The above calculations can also be applied to the case of scalar photons leading to scalar potentials. The Todorov potentials were used by Crater and Van Alstine and collaborators in their spectroscopic calculations [31].

Since expression (9) contains in a compact form leading contributions of multiphoton exchange diagrams, it could also be continued to the nonperturbative domain by formally increasing the value of the fine structure constant $\alpha$. One might in this case get an insight into a strong coupling regime of QED. This study was done through the analysis of the behaviour of the positronium spectrum [43]. There appears a critical value of $\alpha, \alpha_{\mathrm{c}}=1 / 2$, for which a fall to the center phenomenon occurs. The latter can be analyzed with different regularization methods. One of these is the Case self-adjoint extension method [44] which guarantees the orthogonality of states with different energy eigenvalues. The new spectrum displays a zero mass pseudoscalar bound state pointing out the occurrence of a spontaneous breakdown of chiral symmetry [43]. This result is in accordance with the similar results obtained with the Bethe-Salpeter equation with a one-photon exchange [45] and with (noncompact) lattice QED [46,47]. 
Other aspects of three-dimensional reductions of the Bethe-Salpeter equation were analyzed by Bijtebier and Broekaert [48].

In conclusion, the constraint theory, applied to two-particle systems, allows a consistent construction of the Poincaré invariant potential theory. Furthermore, it establishes a bridge between the latter and the quantum field theory providing at the same time a practical means for the resolution of the Bethe-Salpeter equation.

\section{References}

1. Relativistic Action at a Distance: Classical and Quantum Aspects, proceedings of the Barcelona Workshop, 1981, edited by Llosa J. (Lecture Notes in Physics, vol. 162) (Springer, Berlin, 1982).

2. Wheeler J. A. and Feynman R., Rev. Mod. Phys., 1949, vol. 21, p. 425.

3. Dirac P. A. M., Rev. Mod. Phys., 1949, vol. 21, p. 392.

4. Bel L., Ann. Inst. H. Poincaré, 1970, vol. 12, p. 307; Bel L., Salas A. and Sánchez, Phys. Rev. D, 1973, vol. 7, p. 1099; Bel L. and Martin J., Phys. Rev. D, 1973, vol. 8, p. 4347; ibid., 1974, vol. 9, p. 2760; Ann. Inst. H. Poincaré, 1980, vol. 33, p. 409; ibid., 1981, vol. 34, p. 231.

5. Gaida R. P. and Tretyak V. I., Acta Physica Polonica, 1980, vol. B11, p. 509; ibid., p. 523; Gaida R. P., Kluchkovsky Yu. B. and Tretyak V. I., Theor. Math. Phys., 1980, vol. 44, p. 687; ibid., 1980, vol. 45, p. 963; ibid., 1983, vol. 55, p. 372.

6. Dominici D., Gomis J. and Longhi G., Nuovo Cimento,1978, vol. 48A, p. 257; ibid., 1980, vol. 56A, p. 263; Longhi G., Ref. [1], p. 135; Longhi G., Dominici D., Gomis J. and Lobo J. A., Ref. [1], p. 149.

7. Lusanna L., Phys. Rep., 1990, vol. 185, p. 1.

8. Llosa J. A., Marques F. and Molina F., Ann. Inst. H. Poincaré, Phys. Théor., 1980, vol. 32, p. 303; Iranzo V., Llosa J. A., Molina A. and Marques F., Ann. Phys. (N.Y.), 1983, vol. 150, p. 114.

9. Bakamjian B. and Thomas L. H., Phys. Rev., 1953, vol. 92, p. 1300.

10. Foldy L. L., Phys. Rev., 1961, vol. 122, p. 275.

11. Currie D. G., Jordan T. F. and Sudarshan E. C. G., Rev. Mod. Phys., 1963, vol. 4, p. 1470.

12. Kerner E. H., J. Math. Phys., 1965, vol. 6, p. 1218.

13. Pauri M. and Prosperi G. M., J. Math. Phys., 1976, vol. 17, 1468; Sazdjian H., Nucl. Phys., 1979, vol. B161, p. 469.

14. Dirac P. A. M., Lectures on Quantum Mechanics (Belfer Graduate School of Science Monograph Series, Yeshiva University, New York, 1964).

15. Constraint's Theory and Relativistic Dynamics, proceedings of the Firenze Workshop, 1986, edited by Longhi G. and Lusanna L. (World Scientific, Singapore, 1987).

16. Droz-Vincent Ph., Lett. Nuovo Cimento, 1969, vol. 1, p. 839; Phys. Scr., 1970, vol. 2, p. 129; Ann. Inst. H. Poincaré, 1977, vol. 27, p. 407; ibid., 1980, vol. 32A, p. 377.

17. Todorov I. T., Dubna Report No. E2-10125, 1976 (unpublished); Ref. [1], p. 213.

18. Komar A., Phys. Rev. D, 1978, vol. 18, p. 1881; p. 1887; p. 3617.

19. Foldy L. L. and Krajcik R. A., Phys. Rev. D, 1975, vol. 12, p. 1700.

20. Coester F. and Havas P., Phys. Rev. D, 1976, vol. 14, p. 2556; Coester F., Helv. Phys. Acta, 1965, vol. 38, p. 7; Ref. [15], p. 159. 
21. Sokolov S. N., Teor. Mat. Fiz., 1975, vol. 23, p. 355; ibid., 1978, vol. 36, p. 193; Dokl. Akad. Nauk. SSSR, 1977, vol. 233, p. 575; Sokolov S. N. and Shatnii A. N., Teor. Mat. Fiz., 1978, vol. 37, p. 271.

22. Rohlrich F., Phys. Rev. D, 1981, vol. 23, p. 1305.

23. Sazdjian H., Ann. Phys. (N.Y.), 1981, vol. 136, p. 136.

24. Lusanna L., in Extended Objects and Bound Systems, proceedings of the Karuizawa International Symposium, 1992, edited by Hara O., Ishida S. and Naka S. (World Scientific, Singapore, 1992), p. 69; Int. J. Mod. Phys. A, 1995, vol. 10, p. 3531; ibid., p. 3675; Lusanna L. and Valtancoli P., ibid., 1997, vol. 12, p. 4769; hep-th/9705156; hep-th/9707072.

25. Takabayasi T, Prog. Theor. Phys., 1975, vol. 54, p. 563; Prog. Theor. Phys. Suppl., 1979, vol. 67 , p. 1.

26. Leutwyler H. and Stern J., Ann. Phys. (N. Y.), 1978, vol. 112, p. 94; Nucl. Phys., 1978, vol. B133, p. 115; Phys. Lett., 1978, vol. 73B, p. 75.

27. Crater H. W. and Van Alstine P., Ann. Phys. (N.Y.), 1983, vol. 148, p. 57; Phys. Rev. D, 1987, vol. 36, p. 3007.

28. Sazdjian H., Phys. Rev. D, 1986, vol. 33, p. 3401; J. Math. Phys., 1988, vol. 29, p. 1620.

29. Mitra A. N. and Santhanam I, Few Body Systems, 1992, vol. 12, p. 41.

30. Mourad J. and Sazdjian H., J. Math. Phys., 1994, vol. 35, p. 6379.

31. Crater H. W. and Van Alstine P., Phys. Rev., 1988, vol. 37, p. 1982; Crater H. W., Becker R. L., Wong C. Y. and Van Alstine P., Phys. Rev. D, 1992, vol. 46, p. 5117.

32. Bijtebier J., Few Body Systems, 1987, vol. 3, p. 46; Bijtebier J. and Broekaert J., Nuovo Cimento A, 1992, vol. 105, p. 351; ibid., p. 625.

33. Sazdjian H., Ann. Phys. (N.Y.), 1989, vol. 191, p. 52.

34. Droz-Vincent Ph., Phys. Rev. A, 1995, vol. 52, p. 1837.

35. Salpeter E. E. and Bethe H. A., Phys. Rev., 1951, vol. 84, p. 1232; Gell-Mann M. and Low F., ibid., p. 350; Nakanishi N., Suppl. Prog. Theor. Phys., 1969, vol. 43, p. 1.

36. Jallouli H. and Sazdjian H., Ann. Phys. (N.Y.), 1997, vol. 253, p. 376.

37. Logunov A. A. and Tavkhelidze A. N., Nuovo Cimento, 1963, vol. 29, p. 380; Logunov A. A., Tavkhelidze A. N., Todorov I. T. and Khrustalev O. A., ibid., 1963, vol. 30, p. 134; Blankenbecler R. and Sugar R., Phys. Rev., 1966, vol. 142, p. 1051; Gross F., ibid., 1969, vol. 186, p. 1448; Partovi M. H. and Lomon E. L., Phys. Rev. D, 1970, vol. 2, p. 1999; Faustov R. N., Teor. Mat. Fiz., 1970, vol. 3, p. 240 [Theor. Math. Phys., 1970, vol. 3, p. 478]; Fronsdal C. and Huff R. W., Phys. Rev. D, 1971, vol. 3, p. 933; Lepage G. P., Phys. Rev. A, 1977, vol. 16, p. 863; Mandelzweig V. B. and Wallace S. J., Phys. Lett. B, 1987, vol. 197, p. 469.

38. Todorov I. T., Phys. Rev. D, 1971, vol. 3, p. 2351; in Properties of Fundamental Interactions, edited by Zichichi A. (Editrice Compositori, Bologna, 1973), vol. 9, Part C, p. 931.

39. Bethe H. A. and Salpeter E. E., Quantum Mechanics of One- and Two-Electron Atoms (Springer Verlag, Berlin, 1957), p. 193; Berestetskii V. B., Lifshitz E. M. and Pitaevskii L. P., Relativistic Quantum Theory (Pergamon, Oxford, 1971), vol. 4, Part 1, p. 283.

40. Bodwin G. T. and Yennie D. R., Phys. Rep. C, 1978, vol. 43, p. 267.

41. Barbieri R., Ciafaloni M. and Menotti P., Nuovo Cimento, 1968, vol. 55A, p. 701.

42. Love S., Ann. Phys. (N.Y.), 1975, vol. 113, p. 153.

43. Bawin M., Cugnon J. and Sazdjian H., Int. J. Mod. Phys. A, 1996, vol. 11, p. 5303. 
44. Case K. M., Phys. Rev., 1950. vol. 80, p. 797.

45. Miransky V. A. and Fomin, Sov. J. Part. Nucl. Phys., 1985, vol. 16, p. 203.

46. Bartholomew J. et al., Nucl. Phys., 1984, vol. B230, p. 222.

47. Göckeler M. et al., Nucl. Phys., 1992, vol. B371, p. 713.

48. Bijtebier J. and Broekaert J., J. Phys. G, 1996, vol. 22, p. 559; ibid., p. 1727; Nucl. Phys., 1997, vol. A612, p. 279; Bijtebier J., ibid., 1997, vol. A623, p. 498.

\section{Зв'язок потенціяльної теорії з квантовою теорією поля}

\section{Г.Сазджан}

Університет “Париж XI", Інститут ядерної фізики, група теоретичної фізики, Франція

Отримано 12 березня 1998 р.

Теорія в'язей застосовується до двочастинкової системи для побудови Пуанкаре-інваріянтних хвильових рівнянь з потенціялами взаємодії. Останні можуть бути визначені у квантовій теорії поля із Фейнманових діяграм. Їхні властивості проаналізовано у випадку квантової електродинаміки. Теорія в'язей забезпечує послідовний спосіб розв'язування рівняння Бете-Солпітера з коваріянтними пропаґаторами.

Ключові слова: релятивістичні хвильові рівняння, рівняння Бете-Солпітера, зв'язані стани, теорія в'язей

PACS: $03.65 . P m, 11.10 . S t, 11.80 . F v$ 